\title{
Simultaneous side-by-side self-expanding metal stent placement using a two-channel endoscope for a bilioenteric stricture after Child's resection
}

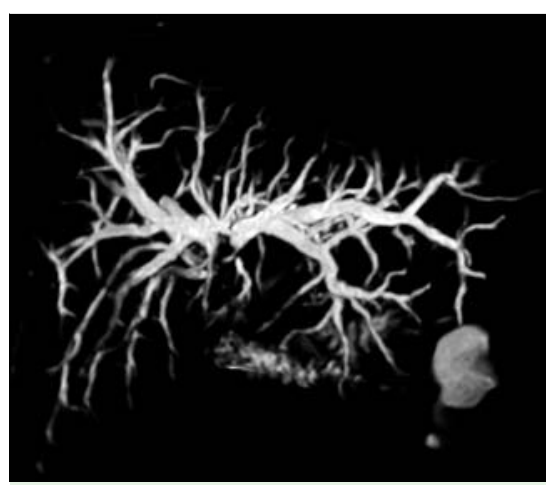

Fig. 1 A three-dimensional magnetic resonance cholangiopancreatography (MRCP) image at the time when the stenosis recurred 14 months after surgery showing diffuse dilatation of the left and right intrahepatic bile ducts and apparent displacement of the left and right bile ducts.

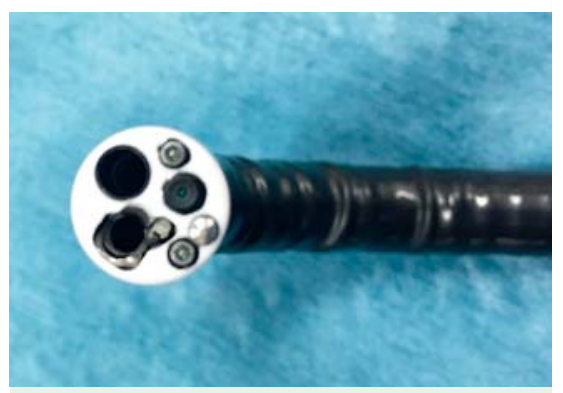

Fig. 2 The two-channel forward-viewing endoscope with an outer diameter of $1.8 \mathrm{~mm}$, a field of view of $120^{\circ}$, a working length of $1030 \mathrm{~mm}$, and two large forceps channels of $2.8 \mathrm{~mm}$ and $3.7 \mathrm{~mm}$, the former having a forceps elevator.

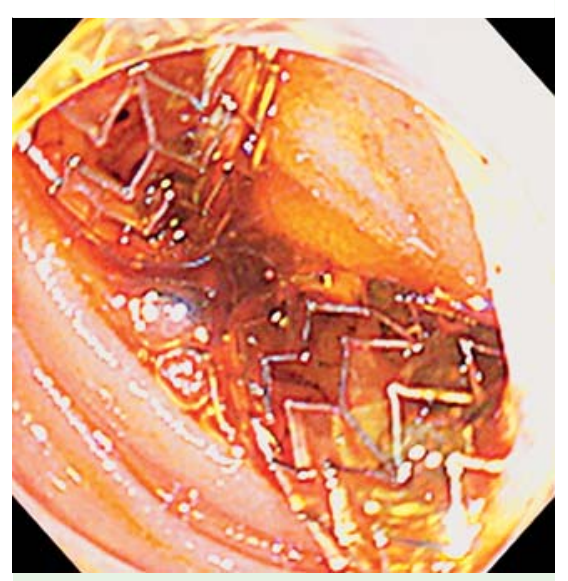

Fig. 3 Endoscopic image taken from the jejunal side after placement of the self-expanding metal stents (SEMSs).

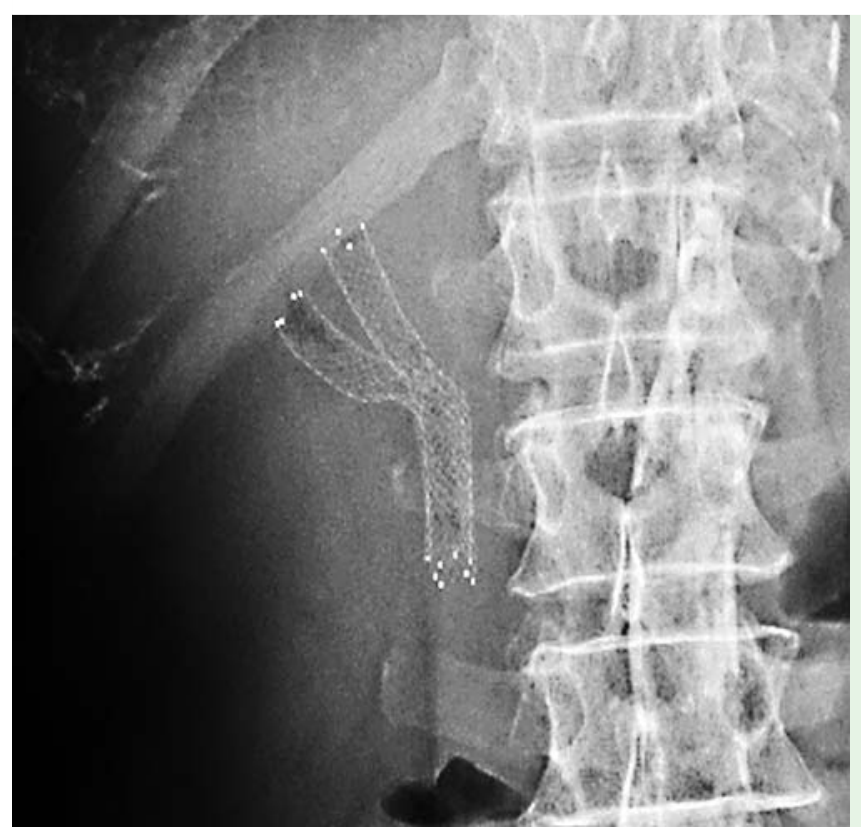

Fig.4 A radiographic image taken on the day after the endoscopic retrograde cholangiopancreatography (ERCP) showing good patency of the two selfexpanding metal stents (SEMSs) in the left and right bile ducts and no stent migration.

Recently, balloon enteroscope-assisted endoscopic retrograde cholangiopancreatography (ERCP) has been attempted to treat malignant choledochojejunal anastomotic stenosis. The development of short-type balloon enteroscopes with large-channel diameter has increased the number of instruments that can be used, allowing complex surgical procedures to be performed [1-3]. However, bilateral biliary drainage is technically very difficult in patients whose right and left bile ducts have been displaced. Patients' quality of life can be markedly compromised if percutaneous transhepatic cholangiodrainage is also required.

We describe a 69-year-old man with extrahepatic bile duct cancer who underwent a Child's resection. Simultaneous side-by-side placement of self-expanding metal stents (SEMSs) was performed using a two-channel endoscope for treatment of his malignant choledochojejunal anastomotic stricture. The malignant stricture had recurred 14 months after surgery, and obstructive jaundice had developed. Fig. 1 shows a three-dimensional magnetic resonance cholangiopancreatography (MRCP) image obtained at the time of the recurrence.
A two-channel endoscope (GIF-2T240; Olympus Medical Systems, Tokyo, Japan) was inserted into the site of choledochojejunostomy, taking 13 minutes ( Fig.2). Guidewires were placed into the left and right bile ducts from each channel. Zilver

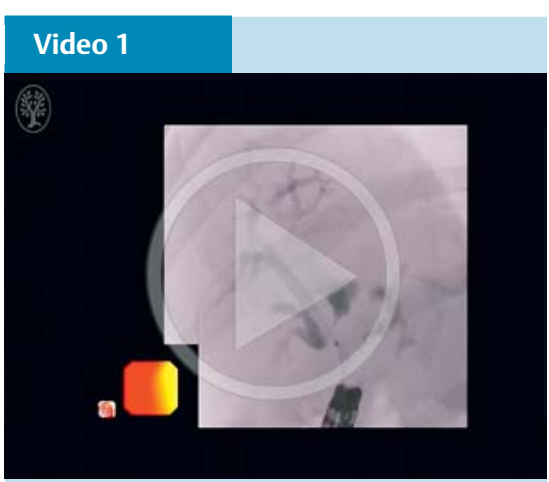

A two-channel forward-viewing endoscope is inserted into the choledochojejunostomy site. A catheter and guidewire are passed through the stricture site and are deeply inserted into the intrahepatic bile duct. Cholangiography shows general displacement of the left and right bile ducts. Guidewires, from each channel, are inserted into the left and right bile ducts. Self-expanding metal stents (SEMSs) are simultaneously placed side-by-side using a 6-Fr delivery system, with the lower ends of the stents being within the jejunum. 
635 biliary SEMSs (8-mm, 6-Fr delivery system; Cook Medical, Tokyo, Japan) were simultaneously placed side-by-side, instead of partially stent-in-stent ( Video 1; $\bullet$ Fig.3), taking 52 minutes. Radiography on the day following stent insertion showed good patency of the two SEMSs ( $\bullet$ Fig. 4), and biliary drainage was promptly performed.

We believe it should be easily possible to perform plastic stent-in-stent placement at the time of re-intervention. Simultaneous side-by-side SEMS placement using a two-channel endoscope is a useful procedure for internal drainage in patients with a Child's resection who require bilateral biliary drainage where this is impossible using a balloon enteroscope.

Endoscopy_UCTN_Code_TTT_1AR_2AZ

Competing interests: None

\section{Kosuke Okuwaki, Hiroshi Yamauchi, Mitsuhiro Kida, Hiroshi Imaizumi, Shiro Miyazawa, Tomohisa Iwai, Wasaburo Koizumi}

Department of Gastroenterology, Kitasato University School of Medicine, Kanagawa, Japan

\section{References}

1 Yamauchi H, Kida M, Okuwaki $K$ et al. Passive-bending, short-type single-balloon enteroscope for endoscopic retrograde cholangiopancreatography in Roux-en-Y anastomosis patients. World J Gastroenterol 2015; 21: 1546-1553

2 Shimatani M, Matsushita M, Takaoka M et al. Effective "short" double-balloon enteroscope for diagnostic and therapeutic ERCP in patients with altered gastrointestinal anatomy: a large case series. Endoscopy 2009; 41: 849-854

3 Shimatani M, Takaoka M, Ikeura T et al. Evaluation of endoscopic retrograde cholangiopancreatography using a newly developed short-type single-balloon endoscope in patients with altered gastrointestinal anatomy. Dig Endosc 2014; 26: 147-155
Bibliography

Dol http://dx.doi.org/

10.1055/s-0042-101384

Endoscopy 2016; 48: E51-E52

(c) Georg Thieme Verlag KG

Stuttgart · New York

ISSN 0013-726X

\section{Corresponding author}

\section{Kosuke Okuwaki, MD}

Department of Gastroenterology

Kitasato University School of Medicine

1-15-1 Kitasato

Minami, Sagamihara

Kanagawa 252-0374

Japan

Fax: +81-42-7788390

kokuwaki@kitasato-u.ac.jp 